\title{
On the location and structure of the artificial 630-nm airglow patch over Sura facility
}

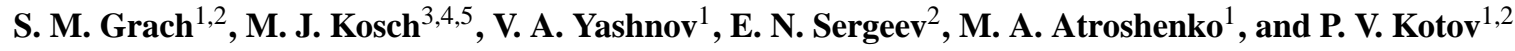 \\ ${ }^{1}$ Radiophysics Faculty, State University of Nizhny Novgorod, Russia \\ ${ }^{2}$ Radiophysical Research Institute, Nizhny Novgorod, Russia \\ ${ }^{3}$ Communication Systems, Lancaster University, Lancaster LA1 4WA, UK \\ ${ }^{4}$ Honorary Research Fellow, University of Kwazulu-Natal, Durban 4001, South Africa \\ ${ }^{5}$ Currently at Space Vehicles Directorate, Air Force Research Laboratory, Hanscom Air Force Base, MA, USA
}

Received: 13 September 2006 - Revised: 2 February 2007 - Accepted: 15 February 2007 - Published: 29 March 2007

\begin{abstract}
Results are presented of the artificial optical emission of the atomic oxygen red line (the radiation of level $\mathrm{O}\left({ }^{1} \mathrm{D}\right)$ with a wavelength of $\left.630 \mathrm{~nm}\right)$ from the HF-pumped ionosphere, obtained in September 2004 at the SURA heating facility situated near Nizhny Novgorod, Russia. For vertical pumping the airglow patch was increasingly displaced to the north, up to $7-8^{\circ}$, with increasing reflection altitude. For large brightness of the emission, the airglow patch started to develop at the northern edge of the pump beam and later expanded to the south. These effects are attributed to the precipitation of supra-thermal electrons from the pump wave upper hybrid resonance altitude to lower altitudes where excitation of the $\mathrm{O}\left({ }^{1} \mathrm{D}\right)$ level is more effective due to the larger density of atomic oxygen, and the $\mathrm{O}\left({ }^{1} \mathrm{D}\right)$ lifetime is shorter. For a pump beam inclination of $12^{\circ}$ to the south, the optical spot was displaced by $4-5^{\circ}$ to the south relative to the straight-line projection of the pump beam onto the sky. This exceeds that expected from the ray tracing and may be related, most probably, to the so-called "magnetic zenith" effect. In addition, mid-scale $(1-10 \mathrm{~km})$ magnetic field-aligned structures were observed in the pumped volume of the ionosphere. The east-west motions of the airglow patches are also analyzed.
\end{abstract}

Keywords. Ionosphere (Active experiments; Wave-particle interactions)

\section{Introduction}

A powerful O-polarized HF radio wave injected into the ionospheric F-region from the ground excites HF plasma waves near and slightly below its reflection point. These waves heat the plasma (a 250-300\% enhancement of the electron temperature can be achieved) and accelerate elec-

Correspondence to: S. M. Grach

(sg@ nirfi.sci-nnov.ru) trons up to energies of 5-30 eV (Carlson et al., 1982; Leyser et al., 2000). Recently, Gustavson et al. (2005) found some evidence of electron acceleration up to $60 \mathrm{eV}$. The plasma heating and electron acceleration cause an airglow enhancement due to excitation of the neutrals by electron impact (Haslett and Megill, 1974). The brightest optical emission is the red line of atomic oxygen (the radiation of level $\mathrm{O}\left({ }^{1} \mathrm{D}\right.$ ) with a wavelength of $630 \mathrm{~nm}$ and has an excitation threshold energy of $1.96 \mathrm{eV}$ ). The other emissions from atomic oxygen and molecular nitrogen $\left(\mathrm{O}\right.$ and $\left.\mathrm{N}_{2}\right)$ at wavelengths $557.7 \mathrm{~nm}$ [level $\left.\mathrm{O}\left({ }^{1} \mathrm{~S}\right)\right], 844.6 \mathrm{~nm}$ [level O $\left.\left(3 \mathrm{p}^{3} \mathrm{P}\right)\right]$ and $427.8 \mathrm{~nm}$ [level $\left.N_{2}^{+}\left(B^{2} \Sigma_{u}^{-}\right)\right]$have larger excitation thresholds $(4.17,10.99$, $18 \mathrm{eV}$, respectively) and smaller excitation cross sections; see, e.g. Gustavson et al. (2005). The artificial airglow has been studied since early 1970s at different ionospheric heating facilities, such as Arecibo (Puerto-Rico), Platteville (Boulder, Colorado), EISCAT (Troms $\varnothing$, Norway), SURA (Nizhniy Novgorod, Russia), HAARP and HIPAS (Alaska). Observations of the artificial airglow are used to study the electron acceleration by HF pump-driven electrostatic plasma waves, as well as for mapping of the ionosphere (Haslett and Megill, 1974; Bernhardt et al., 1989a,b; Leyser et al., 2000; Bernhardt et al., 1991, 2000; Pedersen and Carlson, 2001; Gustavson et al., 2001, 2005; Gumerov et al., 1999; Grach et al., 2004; Kosch et al., 2002a, 2005). Mapping of the pumped volume with CCD cameras is used for remote sensing of the large-scale irregularities of both natural and HF-pump driven origin. Velocities of their drift motion, as well as diffusion coefficients and the lifetime of the $\mathrm{O}\left({ }^{1} \mathrm{D}\right)$ quenching can be determined from an analysis of the artificial airglow images (Bernhardt et al., 1989b; Leyser et al., 2000; Bernhardt et al., 2000; Pedersen and Carlson, 2001). Recently, the so-called magnetic zenith effect (MZE), a significant enhancement of the airglow intensity accompanied by a dramatic increase in the electron temperature during pumping along the geomagnetic field line direction, was discovered with the use of optical observations at the HAARP

Published by Copernicus GmbH on behalf of the European Geosciences Union. 
and EISCAT facilities (Kosch et al., 2000; Gurevich et al., 2002; Pedersen et al., 2003; Rietveld et al., 2003). In some experiments mid-scale $(\sim 1 \mathrm{~km})$ magnetic field-aligned structures in the HF pumped ionosphere are seen in the optical images (see, e.g. Djuth et al., 2005). Such structures were also obtained by different radio methods and in-situ measurements (Basu et al., 1997; Kelley et al., 1995; Frolov et al., 2001; Myasnikov et al., 2001; Djuth et al., 2006). The existence of such structures is supported by theoretical predictions (e.g. Perkins and Valeo, 1974; Vas'kov and Gurevich, 1979; Gurevich et al., 2002).

Two candidates for the electron acceleration are Langmuir waves and upper hybrid turbulence. Langmuir waves appear due to the ponderomotive parametric instabilities near the pump wave reflection point $z_{0}$, at which $f_{\mathrm{pe}}\left(z_{\mathrm{r}}\right)=f_{0}$, and propagate mainly along the magnetic field (Al'ber et al., 1974; Perkins et al., 1974; DuBois et al., 1990). Upper hybrid waves initially appear due to the thermal parametric instability, mainly across the magnetic field, a few kilometers below $z_{\mathrm{r}}$ near the upper hybrid (UH) resonance altitude $z_{\mathrm{UH}}$, at which $f_{\mathrm{pe}}\left(z_{\mathrm{UH}}\right)=\left[f_{0}^{2}-f_{\mathrm{ce}}^{2}\left(z_{\mathrm{UH}}\right)\right]^{1 / 2}$, where $f_{\mathrm{pe}}$ and $f_{\mathrm{ce}}$ are the local ionospheric electron plasma and electron cyclotron frequencies, respectively (Grach et al., 1977; Vas'kov and Gurevich, 1977; Robinson, 1989). During the developed stage of the thermal parametric instability, the upper hybrid waves essentially broaden their spatial and angular spectrum (Grach et al., 1981) and excite, as a pump, daughter upper hybrid, Langmuir, and lower hybrid waves under different ponderomotive nonlinear processes (Grach, 1981; Kuo et al., 1997; Istomin and Leyser, 1998; Mishin et al., 2004). The question of which candidate dominates is under long-standing theoretical discussion (Weistock and Bezzerides, 1974; Gurevich et al., 1985; Grach et al., 1986; Dimant et al., 1992; Grach, 1999; Gurevich et al., 2002; Istomin and Leyser, 2003) but still remains to be resolved. Recent experimental achievements demonstrated that for long ( $\gtrsim 10 \mathrm{~s}$ ), pumping electron acceleration is linked to the upper hybrid turbulence while short ( $\gtrsim 10 \mathrm{~ms}$ ) pump pulses provide an effective acceleration by Langmuir waves (Gumerov et al., 1999; Gustavson et al., 2001; Kosch et al., 2002b; Grach et al., 2004; Gustavson et al., 2005). Notice that Mishin et al. (2004) have shown that lower hybrid waves can efficiently accelerate electrons during HF heating, although to lesser energies than Langmuir waves.

Below we report new results on the mapping of the ionospheric pumped volume in the oxygen red line $(630 \mathrm{~nm}) \mathrm{ob}-$ tained at the SURA heating facility. These experiments were aimed at studying the MZE for mid-latitude conditions: at the SURA facility the magnetic field elevation angle $\left(71.5^{\circ}\right)$ is less than at EISCAT and HAARP $\left(77.2^{\circ}\right.$ and $75^{\circ}$, respectively), therefore conditions for the MZE must be different at the SURA facility (Gurevich et al., 2005). Some evidence of the MZE at the SURA facility has been obtained by radiotomography from satellites (Tereshchenko et al., 2004). In our experiments we have found new evidence of the MZE. In addition, we were able to resolve mid-scale magnetic fieldaligned structures in the pumped volume; analyzed east-west motions of the airglow patches; and we observed a northward displacement of the airglow patch with increasing pump wave reflection height for vertical pumping. A comparison of the optical patch positions along the north-south meridian with ray tracing of the pump wave reveals which electrostatic waves were responsible for the electron acceleration and therefore for the artificial optical emissions.

\section{Experimental data}

The experiment was carried out in September 2004 at the SURA radio facility near Nizhny Novgorod, Russia (geographical coordinates $56.13^{\circ} \mathrm{N}, 46.10^{\circ} \mathrm{E}$, geomagnetic field declination and inclination are $\approx 10.0^{\circ}$ east and $\approx 71.5^{\circ}$, respectively). According to the ionospheric conditions, on 14 September the SURA facility was operated at frequencies close to the fourth electron gyroharmonic (5.275$5.345 \mathrm{MHz})$. On the other days of the campaign $(15,17$ and 18 September) SURA was operated at the lowest available frequency of transmitters, $f_{0}=4.3 \mathrm{MHz}$. At this frequency the effective radiated power is $\approx 150 \mathrm{MW}$. The HF beam width at the Sura facility is $\approx 12^{\circ}$ when pointing in the vertical direction and by about $1-2^{\circ}$ wider for beam dip angles of $12^{\circ}$ and $16^{\circ}$, which were used in the experiment. A square wave pump modulation of $2 \mathrm{~min}$ on, 2 min off, with a period close to the radiative lifetime of $\mathrm{O}\left({ }^{1} \mathrm{D}\right)(107 \mathrm{~s})$, was used.

Optical imaging was performed using a Peltier-cooled front-illuminated bare CCD camera with 16-bit slow-scan read-out (SBIG ST9E). In addition to cooling, the images were binned down to $256 \times 256$ pixels, in order to increase sensitivity and reduce noise. A $\mathrm{f}=50 \mathrm{~mm}$ F0.7 lens, giving $\mathrm{a} \approx 13^{\circ}$ field of view was used. Using the stars, the camera look direction was adjusted to be parallel to the pump beam. The relatively small field of view gives a spatial resolution of $\approx 0.5 \mathrm{~km}$ in the F-layer, suitable for imaging small-scale structures. For the entire campaign, a $630-\mathrm{nm}$ filter was used with 27-s integration and 3-s housekeeping time, giving a 30-s cycle. Calibration of the images into Rayleighs was performed by using the same imager to view the reflected output of a known lamp. Optical observations of the HF pumped ionosphere were accompanied by recording the spectra of the Stimulated Electromagnetic Emissions (SEE), a secondary radiation from the ionospheric pumped volume (Leyser, 2001), with a HP3585 spectrum analyzer. An ionosonde co-located with SURA made soundings every $10 \mathrm{~min}$. However, some of the ionograms obtained during SURA operations could not be analyzed because of strong anomalous absorption of the ionosonde signal during HF pumping.

On 14 and 15 September the HF beam was pointed in the vertical direction. On 14 September the pump frequency changed every $4 \mathrm{~min}$ (from cycle to cycle) by $10 \mathrm{kHz}$ near 
4th electron gyroharmonic. As estimated by the Broad Upshifted Maximum feature's position in the SEE spectra (for details see, e.g. Leyser, 2001), the interaction between the pump wave and plasma occurred near 260-270 km altitude. Weak red line airglow with a maximum intensity of $\sim 5 \mathrm{R}$ against a decreasing background was obtained around 21:2121:45 LT. Neither the structure of the images nor the average airglow intensity showed a clear dependence on the pump wave frequency.

On 15 September the experiment lasted from 21:10 LT till 23:24 LT with the pump wave frequency fixed at $f_{0}=4.3 \mathrm{MHz}$. The data are presented in Figs. 1-3. Figure 1 (top panel) shows the time sequence of 630-nm airglow intensity, spatially averaged over the imager field of view, in Rayleighs. The data have been background subtracted; the intensity is measured relative to a minimum background intensity obtained at 23:02 LT. The square wave represents the HF pump wave on/off cycles. A decrease in the background brightness for 21:10-23:00 LT is due to decreasing sunlight after sunset.

The lower panel of Fig. 1 shows the real reflection height of the HF pump wave, derived from ionograms vs. time. It should be noted that the accuracy of such a derivation is quite low due to strong natural F-spread. For the ionogram analysis we used the lower border of the reflected ionosonde signal, the upper border would have given altitudes by about $30 \mathrm{~km}$ higher. However, the presence of the reflected HF pump wave signal $\left(f_{0}<f_{\mathrm{O}} \mathrm{F} 2\right)$ corresponded to critical frequencies $f_{\mathrm{O}}$ F2 derived from the lower border. The presence of strong F-spread provides evidence for the existence of strong, largescale irregularities of plasma density in the upper ionosphere.

A correlation between pump wave on/off time and airglow intensity increase/decrease is clearly seen in the upper panel of Fig. 1 during the interval 21:10-23:10 LT. The airglow intensity grows during each "pump on" interval ( $2 \mathrm{~min}$ ), decreases during the "pump-off" intervals, somewhat faster during the first minute of no pumping. During the interval 21:10-21:25 LT, the average intensity of artificial airglow was $\sim 15 \mathrm{R}$ above the background. Later, during the interval 21:30-21:40 LT, it increased up to $25 \mathrm{R}$, then noticeably decreased (up to $\sim 10 \mathrm{R}$ ) after 21:44 LT, and further decreased down to $\sim 5 \mathrm{R}$ after 22:12 LT. The reason for the intensity minimum at 22:20-22:24 LT is discussed later. After 23:08 LT the artificial airglow disappeared, together with a dramatic decrease in the SEE intensity, despite that the ionospheric plasma was still overdense according to ionograms, and the reflection of the pump wave was still observed.

A set of false color airglow patch images during one pump cycle (21:26:00-21:30:00 LT) is presented in Fig. 2. The end time of each 27-s integration is shown in the images. North and east are at the bottom and right of each image, respectively. The white circle corresponds to the straight-line projection of the $12^{\circ}$ wide HF pump beam onto the sky, the black arrow shows the direction of the geomagnetic field projection onto the image (inclination $\approx 10^{\circ}$ east). When processing

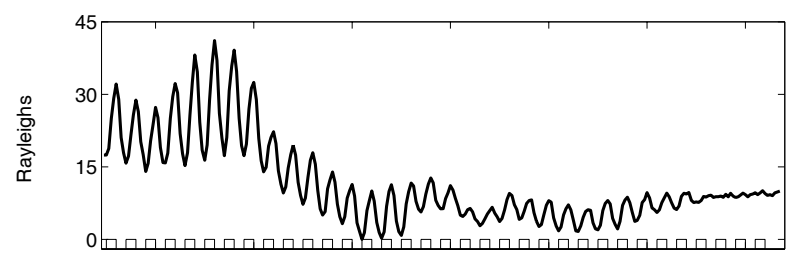

LT

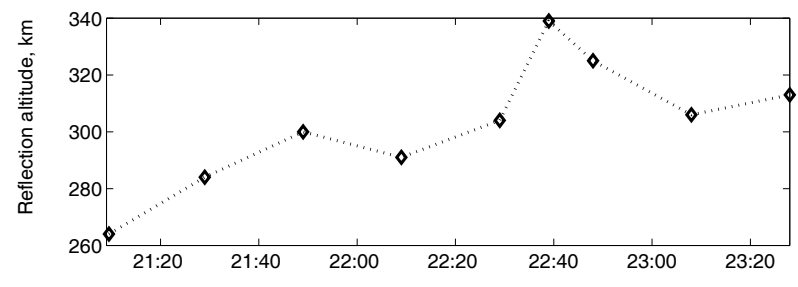

Fig. 1. The average intensity of the 630-nm airglow above the background (top panel). The bars show the pump wave on/off cycle. The bottom panel shows the reflection altitudes of the $O$-polarized pump wave at $4.3 \mathrm{MHz}$ vs. time during the experiment as determined from the ionograms.

the images, we removed bright stars with a $7 \times 7$ median filter, subtracted a dark image (with the end time 21:25:55 LT, just before pump turn on) and performed a running averaging over 11 pixels, both in the vertical and horizontal directions to remove pixel noise. The maximum airglow intensity during this cycle was obtained before the pump switched off (21:27:55 LT) and achieved 42 R (maximum without averaging pixels $=58 \mathrm{R}$ ). The airglow patch started to appear as a double patch: the brighter western portion appeared at the northern edge of the HF beam and expanded later mainly to the south towards the the beam center, along the magnetic field line. The eastern part of the patch appears near the center of the HF beam in the south-north direction and later expands without any displacement. Later, both parts of the patch overlap, which leads to an apparent southwestward expansion of the whole patch. During the pump-off phase the patch does not exhibit any noticeable displacement as it fades. Such a southward spread along the magnetic field, but without a double patch structure and therefore apparent southwestward expansion, was observed only during 4 pump cycles corresponding to a large brightness at the center of the patch $\gtrsim 37-50 \mathrm{R}(21: 26-21: 40 \mathrm{LT})$. In other pump cycles the position of the patches did not vary visibly.

On the other hand, perceptible displacements of the optical patch position over the sequence of images through time were observed. This is illustrated by Fig. 3, which shows a sequence of images obtained during the last $27 \mathrm{~s}$ of each pump-on cycle. Here the same image processing was performed, but the dark images come from integration times just before the corresponding "on" intervals. During 21:10-21:20 LT the patch occupied the central part of the HF beam projection onto the image. Later, at 21:26-21:40 LT, 


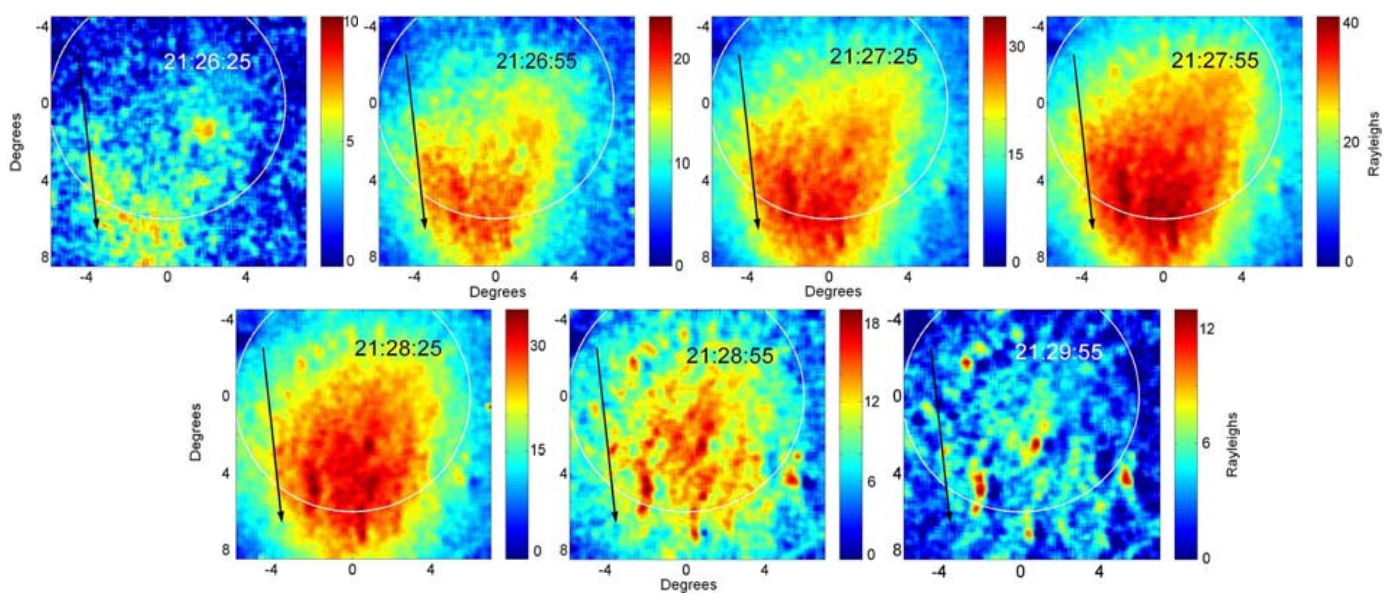

Fig. 2. A sequence of images showing the development and decay of the 630-nm artificial airglow patch during one HF pumping cycle obtained at 21:26-21:30 LT on 15 September 2004. The integration time is $27 \mathrm{~s}$, the end time of the integration is shown in the panels. The images are background subtracted and calibrated into Rayleighs. The background image was recorded at 21:25:55 LT. North and east are at the bottom and right of each image. The white circle is a mapping of the $12^{\circ}$ wide HF pump beam. The black arrows show the direction of the geomagnetic field projection.

simultaneously with an increase of the airglow brightness (Fig. 1), the patch displaces to the north, and the maximum brightness was observed at $\approx 5^{\circ}$ from the center of the HF beam. At the same time the patch displaced to the east by approximately $1^{\circ}$ every $4 \mathrm{~min}$, which corresponds to a horizontal velocity of $\approx 20 \mathrm{~m} / \mathrm{s}$ at an altitude of 280 $300 \mathrm{~km}$. At 21:46-22:06 LT, when the 630-nm airglow intensity decreases, the northward displacement decreased by $\approx 1^{\circ}$, and the patch did not move noticeably. During this time, some optical structures approximately parallel to geomagnetic field projection are seen in the images. Such structures are seen also from time to time in the earlier and later images (e.g. images from 21:23:55 LT and 21:55:55 LT in Fig. 2). During 22:10-22:20 LT, the airglow patch displaced to the north by $\approx 4^{\circ}$, the brightest area displaced from $3-4^{\circ}$ to $7-8^{\circ}$ to the north from the center of the HF beam projection concurrent with westward drifting of the patch. The behavior of subsequent airglow patches looks like successive east-west drifting of 2 blobs, in which the 630-nm airglow is excited by the HF pump, with the same angular velocity of $0.63^{\circ}$ per $4 \mathrm{~min}$ (linear velocity $\approx 13 \mathrm{~m} / \mathrm{s}$ at $280-300 \mathrm{~km}$ ), but with different positions in the south-north direction. The first patch with the brightest area at $\gtrsim 7-8^{\circ}$ to the north from the beam center existed at 22:22-22:36 LT; the second patch at $\approx 4-6^{\circ}$ to the north existed from 22:42 till 23:08 LT. Later, the artificial $630-\mathrm{nm}$ airglow was not excited. This is consistent with a successive motion of the airglow clouds across the ionospheric heated volume reported by Bernhardt et al. (2000).

Three points are noted. First, a noticeable decrease in the average artificial airglow intensity in Fig. 1 at 22:2222:30 LT is due to the fact that about a half of the airglow patch is outside the imager field of view. Second, the median filter removed only the brightest stars, but the weaker ones, appearing about 5-10 R brighter than the background, remain in the images (see images taken from 22:23 till 23:08 LT). It is seen from these images that the artificial airglow patches drift across the imager field of view with the same apparent velocity $\left(0.63^{\circ}\right.$ per $\left.4 \mathrm{~min}\right)$ as the stars. Such westward drift also can be seen at 21:10-21:20 and 21:54-22:20 LT, respectively. Third, in some images (e.g. 21:23:55 LT) two connected airglow patches can be seen. This becomes clear when comparing the images with previous/subsequent images.

The SEE spectra recorded were typical for $f_{0}=4.3 \mathrm{MHz}$, the frequency being situated well between the 3rd and 4th electron cyclotron harmonics and contained prominent Downshifted Maximum, Broad Continuum, and Broad Upshifted Structure spectral features (Frolov et al., 2001; Sergeev et al., 2006). Such type of the SEE spectra are classified as a "strong emission" (Sergeev et al., 2006) and characterize well developed upper hybrid turbulence. The intensity of all features decreased slightly with time till 23:08 LT, after which the intensity rapidly dropped. No clear correlation between the SEE and airglow brightness or patch positions was obtained.

On 17 and 18 September the HF pump beam was inclined to the south of vertical. Unfortunately, the time and quality of the optical measurements were limited by clouds and lasted only $20 \mathrm{~min}$ on both nights. Even during these intervals the sky was covered by a thin cloud layer. The best images obtained are presented in Fig. 4. On 18 September, when the pump beam zenith angle was $16^{\circ}$, the $630-\mathrm{nm}$ airglow patch with a brightness $\lesssim 20 \mathrm{R}$ was located in the center of the HF beam. On 17 September, the HF beam was pointed $12^{\circ}$ south from vertical. In this case, the airglow patch with a brightness 


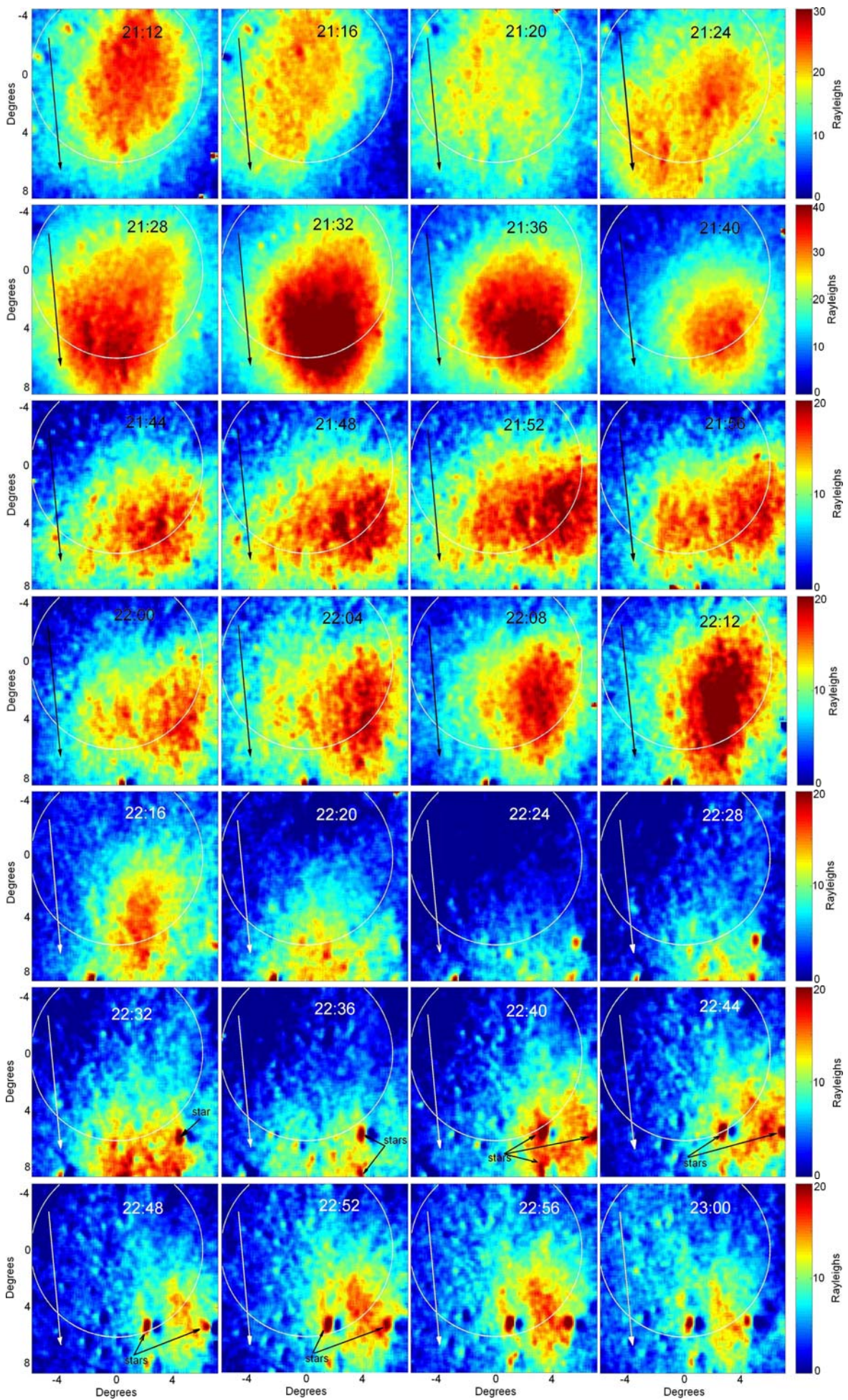

Fig. 3. A collection of images of artificial airglow patches obtained on 15 September 2005. Each panel corresponds to the integration over the last $27 \mathrm{~s}$ of a 2 -min "pump-on" cycle. The end times of each integration $(+5 \mathrm{~s})$ are shown in the panels. The calibration, white circles and arrows are the same as in Fig. 2. North and east are at the bottom and right of each image. 

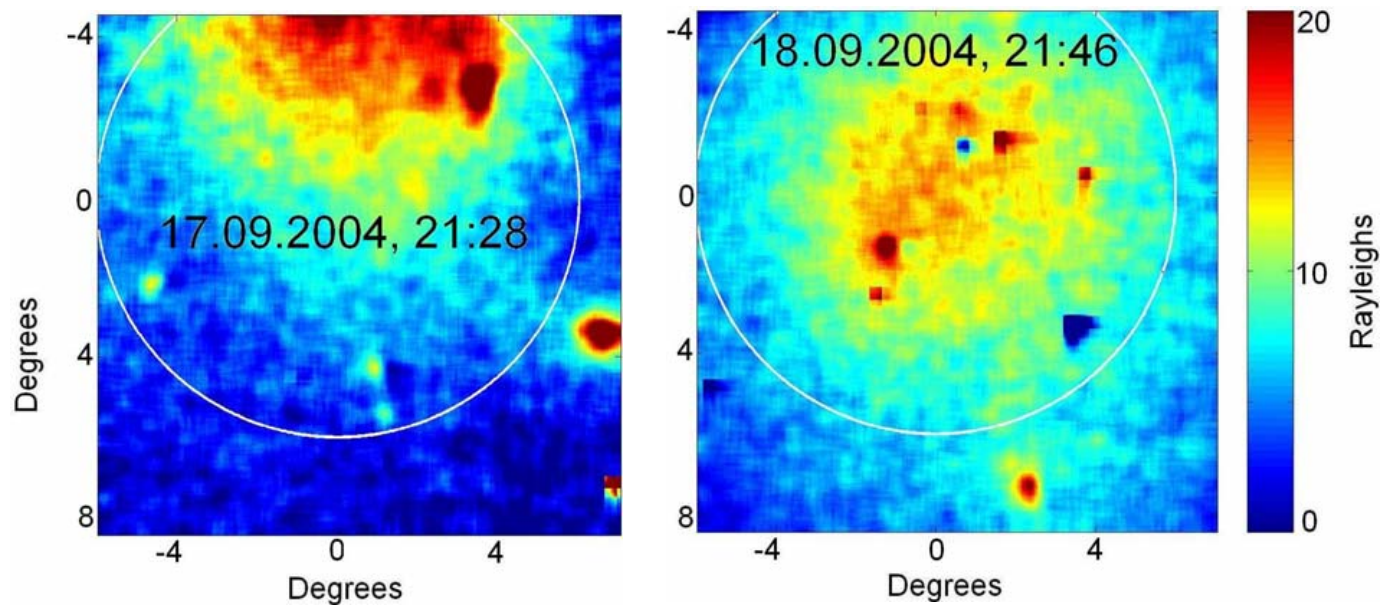

Fig. 4. Images of artificial airglow obtained on 17 September 2004 , for an HF beam zenith angle of $12^{\circ} \mathrm{S}$ (left panel) and on 18 September 2004, for an HF beam zenith angle of $16^{\circ} \mathrm{S}$ (right panel). North and east are at the bottom and right of each image.

$\sim 20 \mathrm{R}$ was clearly shifted to the south from the straight-line projection of the pump beam by $\gtrsim 4^{\circ}$. For this experiment, it is impossible to determine an angular size and position of the southern boundary of the airglow patch, since it extends beyond the imager field of view.

\section{Discussion}

During 14-18 September 2004 we ran a campaign to study the artificial airglow at $630 \mathrm{~nm}$ (the radiation of level $\mathrm{O}\left({ }^{1} \mathrm{D}\right)$ of the atomic oxygen). The airglow appears due to electron acceleration by pump-driven plasma waves and excitation of the neutrals by collisions with the accelerated electrons. The experimental data obtained contributes to the knowledge of the behavior of the pumped volume in the ionosphere. The optical data provides spatial resolution beyond anything a radar can achieve.

\subsection{Variations of the airglow intensity}

The airglow intensity and angular size of the airglow patch varied during the $2.5 \mathrm{~h}$ of experiments on 15 September over rather wide limits: from 5 to $25 \mathrm{R}$ averaged across the imager's field of view, and from 15 to $50 \mathrm{R}$ maximum brightness in the center of the patch. Similar values of the intensity were observed at the SURA facility in 1993 and 1995 by Bernhardt et al. (2000). In the earlier experiments of 1990 during solar maximum, intensities up to $\gtrsim 200 \mathrm{R}$ were observed. Intensities of similar order (20-100 R) have been observed at the Arecibo, EISCAT and HAARP facilities (e.g. Bernhardt et al., 1989a,b; Kosch et al., 2002a; Pedersen and Carlson, 2001). The variations of the brightness do not show any clear correlation with pump power (for the cases where the power exceeds the threshold power for airglow excitation) or with the pump wave frequency, except for the cases of pumping near multiple electron gyrofrequencies, $f_{0} \sim n f_{\text {ce }}, n=2,3,4$, etc. In the latter case a decrease in the brightness was obtained for $n=3$, 4 (Bernhardt et al., 2000; Kosch et al., 2002b) and an increase for $n=2$ (Kosch et al., 2005). In our experiments near the 4th gyroharmonic on 14 September only very weak airglow was obtained. On 15 September a noticeable growth of the brightness (from $\sim 30 \mathrm{R}$ to $\sim 50 \mathrm{R}$ ) occurred, together with an increase in the reflection altitude at $\approx 21: 30$ LT, but later (at about 21:45 LT and about 22:10 LT) reductions in the brightness does not seem to coincide with any other phenomena. The variations of the airglow intensity can be influenced by changes in the background population of suprathermal electrons with energies of $0.5-1 \mathrm{eV}$. According to theoretical studies by Grach et al. (1986); Grach (1999) the efficiency of acceleration strongly depends on the presence and "initial" background of such electrons. This comes about because pump-driven plasma waves do not accelerate thermal electrons effectively. The population of the "initial" suprathermal electrons in the HF-pumped volume of the ionosphere is quite numerous during the daytime but decreases dramatically at nighttime (see, e.g. Sergeev et al., 2004) when optical observations become possible.

Grach et al. (1998) reported that under near-identical ionospheric background conditions (close to local midnight, altitude, critical frequency and scale length of the F-region), and the same pump frequency and power, opposite results were obtained by multi-frequency Doppler sounding of the pumped volume, while on 30 June 1989 a well-defined depletion of ionospheric plasma from the pump wave upper hybrid altitude was observed near midnight and attributed to the plasma heating; an increase in the plasma density related to additional ionization by accelerated electrons occurred on 28-29 August 1990. A weak enhancement of geomagnetic 
activity two days before the latter case was the only difference found. This difference arguably may have contributed to an enhanced background of suprathermal electrons.

In addition, geomagnetic activity reduces the $\mathrm{O} / \mathrm{N}_{2}$ neutral composition in the F-layer through Joule heating. Variations in the $\mathrm{O} / \mathrm{N}_{2}$ ratio up to an order of magnitude are possible (Strickland et al., 2001). The vibrational states of $\mathrm{N}_{2}$ are strong absorbers of pump-accelerated electrons (Mishin et al., 2000; Vlasov et al., 2004), but results in no $\mathrm{N}_{2}$ optical emissions. Hence, any significant geomagnetic activity up to a couple of days prior to an artificial airglow experiment significantly reduces the possibility of producing pump-induced optical emissions. The small airglow intensity on 14 September is possibly due to this effect under the prevailing geophysical conditions $\left(K_{\mathrm{p}}=5+\right)$. On 15,17 and 18 September geophysical conditions were much quieter, namely $K_{p}=2,3$ and 0 , respectively, and the artificial airglow intensity was correspondingly higher.

We suppose that the variations of airglow intensity on 15 September appear to be due to small variations of the suprathermal electron density background, which are very difficult to investigate.

\subsection{Regular northward displacement of the airglow patch}

As was pointed in Sect. 2, a regular displacement of the 630$\mathrm{nm}$ airglow patch to the north by several degrees from the center from the pump beam straight-line projection was observed whilst pumping into the local zenith on 15 September 2004. The displacement appeared during the fourth pump cycle (21:22-21:26 LT), when the western part of the doublepatch was shifted by $\approx 5^{\circ}$ to the north, and existed during all subsequent cycles. The displacement appeared simultaneously with an increase in the pump wave reflection altitude from $260 \mathrm{~km}$ to $280-300 \mathrm{~km}$. The magnitude of the displacement was $\approx 3-5^{\circ}$ during $21: 28-22: 16 \mathrm{LT}$, then increased to $\approx 7-8^{\circ}$, again simultanously with an increase in the reflection altitude up to $\sim 340 \mathrm{~km}$, then decreased to $4-5^{\circ}$, together with a decrease in the reflection altitude down to $310 \mathrm{~km}$.

Two possible explanations appear likely. First, the northward shift of the heated volume may be due to refraction of the O-polarized HF beam according to its group propagation path. Second, precipitation of accelerated electrons along the geomagnetic field from a higher situated acceleration region to lower altitudes where excitation of the $\mathrm{O}\left({ }^{1} \mathrm{D}\right)$ level is more effective due to the larger density of atomic oxygen. In this case the northward inclination of the geomagnetic field would provide the patch displacement.

To examine these possibilities, we performed a ray tracing for the prevailing ionospheric conditions obtained from ionograms during the experiment, and compared it to the optical patch positions. We note again that the accuracy of the ionogram inversion is low due to strong natural F-spread. The results of the raytracing in the magnetic meridian plane are presented in Figs. 5a, b. The solid lines show the paths of the central ray and edge rays $\left( \pm 6^{\circ}\right.$ from the center) of the HF pump beam in the magnetic meridian plane, calculated under the assumption of a horizontally uniform ionosphere, for ionograms obtained at 21:09 LT (panel a) and 22:49 LT (panel b), respectively. Note that there was a systematic shift in the SURA antenna pattern to the south by $\approx 2^{\circ}$ for technical reasons (Yu. V. Tokarev and A. N. Karashtin, private communication). The dotted lines show the straight-line projection of the HF beam onto the sky, the dashed lines correspond to the imager field of view, horizontal lines show the HF beam reflection altitude $z_{\mathrm{r}}$, where $f_{\mathrm{pe}}\left(z_{\mathrm{r}}\right)=f_{0}$, the upper hybrid altitude $z_{\mathrm{UH}}$, where $f_{\mathrm{pe}}\left(z_{\mathrm{UH}}\right)=\left[f_{0}^{2}-f_{\mathrm{ce}}^{2}\left(z_{\mathrm{UH}}\right)\right]^{1 / 2}$, and the arrows show the geomagnetic field direction. The schematic blobs in the panels correspond to the airglow patches obtained at 21:11:55 LT (panel a) and 22:47:55 LT (panel b), respectively. When drawing the airglow blobs we assumed an altitude range of $225-275 \mathrm{~km}$. The airglow blob position in the south-north direction are shown in accordance with the images, and include the area where the airglow brightness exceeds the average. In panel (a), where $z_{\mathrm{r}} \approx 260 \mathrm{~km}$, the patch is situated approximately in the center of the HF beam at the upper hybrid resonance altitude. At the reflection altitude, the center of the patch is shifted by $\approx 10 \mathrm{~km}\left(2^{\circ}\right)$ to the south from the central ray due to refraction of the beam near reflection. The deviation is small (almost straight-line propagation) on the southern edge of the beam; it is $\approx 10 \mathrm{~km}$ in the central part and $\approx 20 \mathrm{~km}$ on the northern edge of the beam at the reflection altitude. In panel (b), where the ionospheric reflection altitude is much higher, the deviation increases by $\approx 2$ times. The projection of the optical patch onto the reflection altitude $z_{\mathrm{r}}$ is slightly (by $\approx 6 \mathrm{~km}$ or $1^{\circ}$ ) shifted to the north from the HF beam central ray, while the northward shift at $z_{\mathrm{UH}}$ is much larger, i.e. $\approx 20 \mathrm{~km}$ or $4^{\circ}$. At first glance this shows that the waveplasma processes occur near the reflection point, namely excitation of Langmuir waves or cavitons under parametric decay or oscillating two-stream instability (DuBois et al., 1990) provide sufficient acceleration of electrons (Gurevich et al., 1985), which excites the oxygen atoms in the immediate vicinity.

However, it is now commonly accepted that the optical emission source has a maximum at altitudes below $z_{\mathrm{r}}, z_{\mathrm{UH}}$, the altitude of the plasma wave excitation and, therefore, electron acceleration, when $z_{\mathrm{r}}, z_{\mathrm{UH}} \gtrsim 270-300 \mathrm{~km}$. Model calculations for the Arecibo heating facility (geographical coordinates $18.48^{\circ} \mathrm{N}, 66.67^{\circ} \mathrm{W}$, geomagnetic field inclination $49^{\circ}$ ) by Bernhardt et al. (1989a), performed for the source altitude of suprathermal electrons from 300 to $400 \mathrm{~km}$ found that the red line airglow should come from the 270to $280-\mathrm{km}$ altitude, while for a source at $250 \mathrm{~km}$ the accelerated electrons produce the excited states of the neutral atomic oxygen locally. Using multi-station observations, Gustavson et al. (2001) found the maximum optical emission to be $5-25 \mathrm{~km}$ below the pump wave reflection altitude. 
Mid-latitude altitude estimates by triangulation Haslett and Megill (1974) show that the optical emission was limited in height to $280 \pm 15 \mathrm{~km}$, although the pump wave reflection height could be up to $30 \mathrm{~km}$ higher. Kosch et al. (2002a) estimated the altitude of the airglow generation to be $\gtrsim 200$ $220 \mathrm{~km}$, while the reflection and upper hybrid altitudes were $30-50 \mathrm{~km}$ higher. Using this information, we place schematically the airglow blob at $225-270 \mathrm{~km}$ altitude. Additional arguments for the placement of the blob below the interaction region are stated in Sects. 3.3 and 3.4. The downward shift of the optical emission source from the pump-plasma interaction altitude (either reflection or upper hybrid altitudes) is conditioned by precipitation of the accelerated electrons along the geomagnetic field to lower altitudes with a higher density of atomic oxygen.

If we project the optical blob along the geomagnetic field line in panel (b) of Fig. 5, it will coincide approximately with the central part of the HF beam at the upper hybrid resonance altitude $z_{\mathrm{UH}}$. On the other hand, a magnetic fieldaligned projection of the central part of the HF beam, which intersects with the reflection level, down to the altitude of the schematic blob, will be shifted by $10-40 \mathrm{~km}\left(2-10^{\circ}\right)$ further to the north from the center of the visible patch position. The same is true qualitatively for other pump cycles with a northward displacement of the patch. Therefore, if we assume the airglow to be generated mainly at the $240-270 \mathrm{~km}$ altitude, we can conclude that electron acceleration occurs mainly near $z_{\mathrm{UH}}$, the upper hybrid resonance of the pump wave. For the ionogram inversion we used the lower boundary of the ionogram trace. This gives the least northward deviation of the ray group path. Any other choice of the trace gives larger ray deviation, a larger northward shift of the reflection point and, therefore, a larger northward shift of the airglow patch, if we assume that electrons precipitate from the reflection altitude. Similar joint analysis of ray tracing and the closest images for other pump cycles led to the same conclusion. Finally, the SEE temporal evolution and stationary spectra measured during the experiment were typical for ones related to upper hybrid turbulence (Sergeev et al., 1999, 2006).

A northward elongation and northward displacement of the airglow patch center by $\approx 30 \mathrm{~km}$ from the source of the accelerated electrons was observed at the Arecibo facility and attributed to an acceleration region from a higher altitude (Bernhardt et al., 1989b). The magnetic field inclination angle is larger at the SURA facility $\left(71.5^{\circ}\right)$ than at Arecibo $\left(49^{\circ}\right)$. This will give larger displacements at Arecibo, than at SURA, while our experiments show similar values. This implies that the vertical displacement is less at Arecibo than SURA. A northward displacement of the airglow patch at mid-latitudes was noted by Adeishvili et al. (1978). However, they did not present any detailed analysis of the data and attributed the displacements to the group propagation of the pump wave (our first option), which is hardly correct, according to our consideration.

\subsection{Temporal evolution of the airglow patches}

The positions of the optical patches did not vary spatially, neither during the growth ("pump-on") nor during the decay ("pump-off") phases for the majority of the pump cycles (30 out of 34 in total). However, during 4 cycles, from 21:26 LT to 21:40 LT, with the largest brightness in the center of the patch (Figs. 1, 3), the airglow patch started to appear at the northern edge of the HF beam and subsequently expanded mainly to the south, towards the center of the beam (see Fig. 2). This phenomenon can be attributed to deeper penetration of the precipitating suprathermal electrons to lower atmosphere layers, down to $220-230 \mathrm{~km}$. Indeed, a stationary distribution of the suprathermal electrons through all altitudes below the source $(z \sim 225-300 \mathrm{~km})$ must be formed during the time $\tau \sim 1 / \delta(\varepsilon) v(\varepsilon) \sim 10-15 \mathrm{~s}$ after the pump is switched on (Gurevich et al., 1985). Here $\delta(\varepsilon)$ is the part of the electron energy lost by one collision, $v(\varepsilon)$ is the electron collision frequency, and $\varepsilon$ is the electron energy. According to Sergeev et al. (1999), the characteristic time of the source (i.e. accelerating HF plasma waves) development is faster than $\tau$ for the pump cycle used, but the image exposure time used is longer than $\tau$. Therefore, already during the first image after the pump is switched on, the airglow generation should occur under a stationary distribution of suprathermal electrons. The larger (by 10-30 R) brightness obtained during these 4 cycles definitely indicates the presence of a larger density of suprathermal electrons in these cycles. Since the northward displacement is attributed to the downward precipitation of the fast electrons from the source along the geomagnetic field line, the appearance of the patch in the first image at $\approx 5-6^{\circ}$ from the center of the HF beam (upper left panel of Fig. 2), and by $\approx 2-3^{\circ}$ after $2 \mathrm{~min}$ of pumping, means that the airglow registered in the first image should occur 10-20 km below that in the central part of the last image during the "pump-on" cycle. According to Gustavson et al. (2001), Fig. 8, the lifetime of the excited $\mathrm{O}\left({ }^{1} \mathrm{D}\right)$ (including quenching by molecular oxygen $\mathrm{O}_{2}$ and nitrogen $\left(\mathrm{N}_{2}\right)$, ambient electrons and atomic oxygen $\left.(\mathrm{O})\right)$ is about $30 \mathrm{~s}$ at $\sim 220-230 \mathrm{~km}$ altitude, increasing with the altitude. This means that the airglow should appear faster at lower altitudes, (i.e. further to the north), while later, when approaching the steady state, the maximum brightness should correspond to the maximum density of the excited $\mathrm{O}\left({ }^{1} \mathrm{D}\right)$, i.e. higher altitudes $(250-270 \mathrm{~km})$ and, therefore, smaller northward displacements. During the relaxation phase ("pump off" time), the brightness should decrease, together with the maximum density of the excited $\mathrm{O}\left({ }^{1} \mathrm{D}\right)$, i.e. at the same altitudes $\sim(250-270) \mathrm{km}$, and, therefore, without any visible displacement of the patch. During the cycles with smaller brightness, the density of the accelerated suprathermal electrons at the low altitudes $\sim 220-230 \mathrm{~km}$ is, most probably, not sufficient to produce any visible effect for the camera used in our experiment. This issue can be better resolved by using an imager capable of higher temporal resolution. 

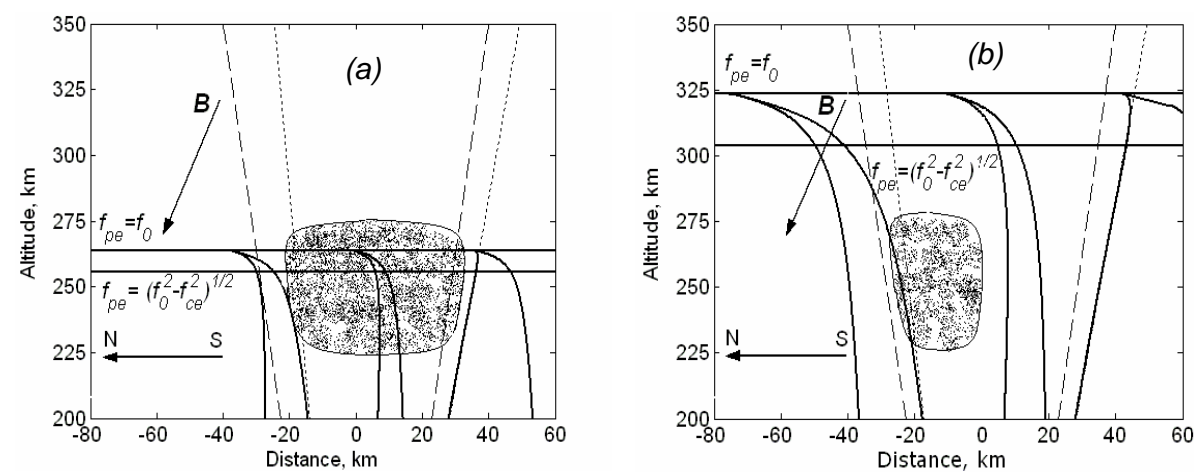

Fig. 5. Ray tracing for the pump wave with $f_{0}=4.3 \mathrm{MHz}$ in the magnetic meridian plane for ionograms, obtained at 21:09 LT (a) and 22:48 (b). Dashed and dotted lines show the imager field of view and the pump beam borders, respectively. Magnetic field direction is shown by arrows, schematic blobs in the panels correspond to the airglow patches obtained at 21:11:55 LT (panel a) and 22:47:55 LT (panel b), respectively.

\subsection{Mid-scale field-aligned structures in the patches}

In some images in Fig. 3, structures approximately parallel to the geomagnetic field line projection are seen. They look like $0.2-1^{\circ}$ wide strips, $10-20 \%$ brighter than neighbouring areas with an angular length of about $3-4^{\circ}$ along the magnetic field projection onto the horizontal plane, and can be seen $\sim 1.5$ min after "pump on" until $\sim 1$ min after "pump off" (see Fig. 2). Similar structures were observed in optical observations by Djuth et al. (2005) at HAARP and Sentman (private communications) at HIPAS. The field-aligned optical enhancements exist due to the enhanced density of the $\mathrm{O}\left({ }^{1} \mathrm{D}\right)$, and therefore inhomogeneities also exist in the spatial distribution of suprathermal electrons. According to our data, the scales of the inhomogeneities across and along the geomagnetic field can be estimated as $l_{\perp} \sim\left[z / \tan (0.2-1)^{\circ}\right\} 1-$ $10 \mathrm{~km}$ and $l_{\|} \sim z /\left[\tan (3-4)^{\circ} \cos \left(71.5^{\circ}\right)\right] \sim 40-50 \mathrm{~km}$, respectively, where $z$ is the altitude of the airglow generation, and the angle $71.5^{\circ}$ corresponds to the geomagnetic field declination. According to Vas'kov and Gurevich (1979); Gurevich et al. (2005), inhomogeneities (depletions) of plasma density with such "mid-range" scale sizes ${ }^{1}$ appear due to self-focusing of the pump wave: the HF waves focus inside the depletions, giving an increase in the pump wave power density and an enhanced level of the HF plasma waves, enhanced plasma heating, and, therefore, further plasma expulsion from the depletions, etc. The depletions are stretched since plasma diffusion and thermal conductivity across the magnetic field are strongly suppressed. In view of the above, more effective electron acceleration, as well as precipitation of the energetic electrons along the geomagnetic field line, should also occur inside the depletions.

\footnotetext{
"Small-scale" irregularities, or striations, usually mean stretched depletions with $l_{\perp} \sim 0.5-50 \mathrm{~m}$.
}

\subsection{East-west variations of the patch positions}

Large-scale irregularities $\left(l_{\perp} \gtrsim 10 \mathrm{~km}\right)$ of both natural and heater-induced origin should also provide focusing of the HF waves in the underdense plasma and guide the HF beam. Hence, the airglow patches follow the motion of the largescale irregularities. By using airglow images we can follow the development of such large-scale structures and the internal motions of the irregularities in the pumped volume of the ionosphere (Bernhardt et al., 1989a,b).

The reasons for the regular northward displacement of the airglow patches and southward expansion of the patches during pumping were discussed above. The east-west motion of the airglow patches have been discussed in several papers (Bernhardt et al., 1989a,b, 2000; Kosch et al., 2000, 2002a). According to Bernhardt et al. (1989a), the typical plasma velocities obtained from both radar and optical observations above the Arecibo heating facility, in January and February of 1987, were westward with speeds of about $20 \mathrm{~m} / \mathrm{s}$. Usually the zonal plasma drift did not vary more than $\pm 30 \mathrm{~m} / \mathrm{s}$ about this value. At the Arecibo facility the star background moves westward with a speed of $20 \mathrm{~m} / \mathrm{s}$ due to the Earth's rotation. In some observations, however, an eastward drift with speeds up to $60 \mathrm{~m} / \mathrm{s}$ were obtained. Bernhardt et al. (2000) reported the eastward drift of the airglow patches obtained on 21 March 1993 above the SURA facility with a linear velocity $\sim 70 \mathrm{~m} / \mathrm{s}$, increasing up to $120 \mathrm{~m} / \mathrm{s}$ during $2 \mathrm{~min}$. Unfortunately, data on drift velocities during other phases of the experiment were not shown in Bernhardt et al. (2000). Kosch et al. (2000, 2002a) found no zonal drift motion of the airglow patches above the EISCAT heater. On 15 September 2004, during several time intervals, namely 21:10-21:20, 21:54-22:20, 22:22-22:36 and 22:42-23:08 LT, the position of the airglow patches drift to the west with the angular velocity of $\approx 0.63^{\circ}$ per 4 min (linear velocity $\approx 13 \mathrm{~m} / \mathrm{s}$ at 280 $300 \mathrm{~km}$ ), i.e. together with star motion across the imager field of view due to the Earth's rotation. During 22:10-22:20 LT 
the westward drift was accompanied by a northward displacement. On the contrary, during 21:26-21:40 LT, an eastward displacement of the patches by $\approx 1^{\circ}$ every 4 min was observed (between successive "pump on" cycles), which corresponds to a linear velocity of $\approx 20 \mathrm{~m} / \mathrm{s}$. However, east-west displacements were not observed for all 2-min "pump-on" times. As a result, we can state that in most cases the largescale plasma structures (in which airglow patches are excited during the pumping cycles) exhibit westward drift, with the same speed as the celestial sphere (star background). This can be easily understood if the large-scale structures do not rotate together with the Earth.

\subsection{Inclined pumping}

Our experiments with the HF beam inclined to the south were aimed to check for the presence of the magnetic zenith effect, obtained at the HAARP and EISCAT facilities (Kosch et al., 2000; Gurevich et al., 2002; Pedersen et al., 2003; Rietveld et al., 2003), at lower latitudes under SURA facility conditions. The amount of data obtained was limited by the clouds. For the successful pump cycles, for both zenith angles of the HF beam $\left(12^{\circ}\right.$ and $16^{\circ},+2^{\circ}$ southward systematic technical shift) the maximum brightness within the airglow patches was observed at the same angle, namely $18^{\circ}-19^{\circ}$ south of vertical, i.e. practically straight along the magnetic field line direction. Ray tracing performed for the ionograms obtained on 17 September shows that the central ray of the HF beam is displaced to the south, compared to the straight projection, by $\sim 1^{\circ}$ at the upper hybrid resonance altitude, and by $\sim 2^{\circ}$ at the maximum altitude of the ray path, i.e. less than the displacement of the patch's brightest area. Unfortunately, it was impossible to perform ray tracing for 18 September due to strong natural F-spread. To estimate the position of the patch and the HF beam path, we used the ionograms from 17 September. The patch's brightest area appeared slightly shifted to the north in comparison to the central ray crossing the upper hybrid resonance altitude. Detailed study of the airglow patch structure for the inclined pumping requires further experiments. The appearance of large-scale structures in the magnetic field line direction was obtained by Tereshchenko et al. (2004), using satellite tomography methods which they claimed to be consistent with the theory of the magnetic zenith effect (Gurevich et al., 2002, 2005).

\section{Conclusions}

Observations of the HF-induced 630-nm airglow performed at the SURA facility in September 2004 have shown the following.

1. A northward displacement of the airglow patch occurs, increasing with the increase of the pump wave reflection altitude. The position of the patch can be explained consistently, if we assume that the electrons, responsible for the $\mathrm{O}\left({ }^{1} \mathrm{D}\right)$ excitation, are accelerated near the upper hybrid altitude of the pump wave and then precipitate at altitudes of $240-270 \mathrm{~km}$.

2. For pump cycles with the largest emission brightness in the center of the patch, the airglow started to appear at the northern part of the HF beam and expanded later, mainly to the south, towards the center of the beam. This can be attributed to a deeper penetration of the precipitating suprathermal electrons to lower atmosphere layers, down to altitudes of $220-230 \mathrm{~km}$, where the lifetime of the excited $\mathrm{O}\left({ }^{1} \mathrm{D}\right)$ decreases noticeably (till about $30 \mathrm{~s}$ ) in comparison with the radiative lifetime.

3. For some cycles, structures approximately parallel to the geomagnetic field projection, corresponding to inhomogeneities (depletions) of plasma density, with scales of $1-10 \mathrm{~km}$ across and $40-50 \mathrm{~km}$ along the geomagnetic field, are seen in the images.

4. In most cases, the large-scale plasma structures within the airglow patches $(>10 \mathrm{~km})$ exhibited a westward drift with the same speed as the coelosphere (star background). However, a slightly faster eastward drift ( $\sim 20 \mathrm{~m} / \mathrm{s}$ ) occurred during $\sim 15 \mathrm{~min}$, together with an increase in the reflection altitude.

5. For $12^{\circ} \mathrm{S}$ and $16^{\circ} \mathrm{S}$ zenith angles of the HF beam, the maximum brightness within the airglow patches was observed to be practically straight along the magnetic field line, namely $18-19^{\circ}$ to the south of vertical.

Acknowledgements. The work was supported by INTAS grant no. 03-51-5583, RFBR grants no. 06-02-17334 and 04-02-17544. The authors are grateful to M. Grill for technical support of the optical equipment and to G. P. Komrakov for support with operating SURA facilty.

Topical Editor M. Pinnock thanks E. Mishin and another referee for their help in evaluating this paper.

\section{References}

Adeishvili, T. G., Gurevich, A. V., Lyakhov, S. B., Managadze, G. G., Milikh, G. M., and Shlyuger, I. S.: Ionospheric emission caused by an intense radio wave, Sov. J. Plasma Phys., 4, 721726, 1978.

Al'ber, Ya. I., Krotova, Z. N., Mityakov, N. A., et al.: Effects of stimulated scattering for an electromagnetic pulse incident on a plasma layer, Sov. Phys. JETP, 39, 275-277, 1974.

Basu, S., Costa, E., Livingston, R. C., Groves, K. M., Carlson, H. C., Chaturvedi, P. K., and Stubbe, P.: Evolution of subkilometer scale ionospheric irregularities generated by high-power HF waves, J. Geophys. Res., 102, 7469-7476, 1997.

Bernhardt, P. A., Tepley, C. A., and Duncan, L. M.: Airglow Enhancements Associated With Plasma Cavities Formed During Ionospheric Heating Experiments, J. Geophys. Res., 94, 90719092, 1989a. 
Bernhardt P. A., Duncan, L. M., and Tepley, C. A.: Heater-induced cavities as optical tracers of plasma drifts, J. Geophys. Res., 94, 7003-7010, 1989b.

Bernhardt, P. A., Scales, W. A., Grach, S. M., Karashtin, A. N., Kotik, D. S., and Polyakov, S. V.: Excitation of artificial airglow by high power radio waves from the "Sura" ionospheric heating facility, Geophys. Res. Lett., 18, 1477-1480, 1991.

Bernhardt, P. A., Wong, M., Huba, J. D., Fejer, B. G., Wagner, L. S., Goldstein, J. A., Selcher, C. A., Folov, V. L., and Sergeev, E. N.: Optical remote sensing of the thermosphere with HF pumped artificial airglow, J. Geophys. Res., 105, 10 657-10 671, 2000.

Carlson, H. C., Wickwar, V. B., and Mantas, G. P.: Observation of fluxes of suprathermal electrons accelerated by HF excited instabilities, J. Atmos. Terr. Phys., 44, 1089-1100, 1982.

Dimant, Ya. S., Gurevich, A. V., and Zybin, K. P.: Acceleration of Electrons in the Ionosphere Under the Action of Intense Radio Wave Near Electron Cyclotron Harmonics, J. Atmos. Terr. Phys., 54, 435-436, 1992.

Djuth, F. T., Pedersen, T. R., Gerken, E. A., Bernhardt, P. A., Selcher, C. A., Bristow, W. A., and Kosch, M. J.: Ionospheric Modification at Twice the Electron Cyclotron Frequency, Phys. Rev. Lett., 94, 125001, doi:10.1103/PhysRevLett.91.12501, 2005.

Djuth, F. T., Reinisch, B. W., Kitrosser, D. F., Elder, J. H., Lee, S. A., and Sales, G. S.: Imaging HF-induced large-scale irregularities above HAARP, Geophys. Res. Lett., 33, L04107, doi:10.1029/2005GL024536, 2006.

DuBois, D. F., Rose, A. H., and Russel, D.: Excitation of strong Langmuir turbulence in plasmas near critical density: Aplication to HF heating of the ionosphere, J. Geophys. Res., 95, $21221-$ $21272,1990$.

Frolov, V. L., Sergeev, E. N., Ermakova, E. N., Komrakov, G. P., and Stubbe, P.: Spectral features of stimulated electromagnetic emissions, measured in the $4.3-9.5 \mathrm{MHz}$ pump wave frequency range, Geophys. Res. Lett., 28, 3103-3106, 2001.

Frolov, V. L., Chugurin, V. V., Komrakov, G. P., Mityakov, N. A., Myasnikov, E. N., Rapoport, V. O., Sergeev, E. N., Uryadov, V. P., Vybornov, F. I., Ivanov, V. A., Shumaev, V. V., Nasyrov, A. M., Nasyrov, I. A., and Groves, K. M.: Study of large-scale irregularities generated in the ionospheric F-region by high-power HF waves, Izv. vuzov. Radiofizika, 43, no 6, 497-519, 2000 (Radiophys. Quantum Electron., Engl. Transl. 43, no. 6, 2000.)

Grach, S. M., Karashtin, A. N., Mityakov, N. A., Rapoport, V. O., and Trakhtengertz, V. Yu.: Parametric interaction between electromagnetic radiation and ionospheric plasma, Radiophys. Quantum Electron., Engl. Transl., 20, 1254-1259, 1977.

Grach, S. M., Mityakov, N. A., Rapoport, V. O., and Trakhtengertz, V. Yu.: Thermal parametric turbulence in a plasma, Physica, 2D, 102-106, 1981.

Grach, S. M.: Electromagnetic radiation from artificial ionospheric plasma turbulence, Radiophys. Quantum Electron., 28, 470-477, 1985.

Grach, S. M., Mityakov, N. A., and Trakhtengertz, V. Yu., Electron acceleration and additional ionization at the parametric plasma heating, Fizika plazmy, 12, 693-702, 1986, in Russian, (Sov. Plasma Phys., Engl. Transl., 12, no. 6, 1986).

Grach, S. M., Komrakov, G. P., Shvarts, M. M., and Yurishchev, M. A.: On frequency dependence of probe wave abnormal attenuation prohile modifying the ionosphere by powerful radio emis- sion, Izv.VUZov - Radiofizika, 41, 966-977, 1998, in Russian (Radiophys. Quantum Electron., Engl. Transl., 41, no. 8, 1998).

Grach, S. M.: On kinetic effects in the ionospheric F-region modified by powerful radio waves, Radiophys. Quantum Electron. Engl. Transl., 42, 572-588, 1999.

Grach, S. M., Sergeev, E. N., Nasyrov, A. M., Gumerov, R. I., Shaimukhametov, R. R., Nasyrov, I. A., and Komrakov, G. P.: Simultaneous observations of the $557.7 \mathrm{~nm}$ airglow and stimulated electromagnetic emission during HF pumping of the ionosphere with diagnostic schedule: first results, Adv. Space Res., 34, 2422-2427, 2004.

Gumerov, R. I., Kapkov, V. B., Komrakov, G. P., and Nasyrov, A. M.: Artificial ionospheric glow caused by short-term effect of highpower RF radiation, Radiophys. Quantum Electron, 42, 463-465, 1999.

Gurevich, A. V., Dimant, Ya. S., Milikh, G. M., and Vas'kov, V. V.: Multiple acceleration of in the region of high-power radio-wave reflection in the ionosphere, J. Atmos. Terr. Phys., 47, 10571070, 1985.

Gurevich, A. V., Zybin, K. P., Carlson, H. C., and Pedersen, T.: Magnetic zenith effect in ionospheric modification, Phys. Lett., A305, 264-274, 2002.

Gurevich, A. V., Zybin, K. P., and Carlson, H. C.: Magnetic-Zenith Effect, Radiophys. Quantum Electron., Engl. Transl., 48, 686699, 2005.

Gustavsson, B., Sergienko, T., Rietveld, M. T., Honary, F., Steen, Å, Brändsröm, B. U. E., Leyser, T. B., Aruliah, A. L., Aso, T., Ejiri, M., and Marple, S.: First Tomographic estimate of volume distribution of HF-pump enhanced airglow emission. J. Geophys. Res., 106, 29 105-29 123, 2001.

Gustavson, B., Sergienko. T., Kosch, M. J., Rietveld M. T., Brändsröm, B. U. E., Leyser, T. B., Isham B., Gallop, P., Aso, T., Ejiri, M., Grydeland, T., Steen, A., LaHoz, C., Kalia, K., Jussia, J., and Holma, H.: The electron energy distribution during HF pumping, a picture painted with all colors, Ann. Geophys., 23, 1747-1754, 2005, http://www.ann-geophys.net/23/1747/2005/.

Haslett, S. C. and Megill, L. R.: A model of enhanced airglow excited by RF radiation, Radio Sci., 9, 1005-1020, 1974.

Istomin, Y. N. and Leyser, T. B.: Parametric interaction of selflocalized upper hybrid states in quantized plasma density irregularities, Phys. Plasmas, 5, 921-931, 1998.

Istomin, Ya. N. and Leyser, T. B.: Electron acceleration by cylindrical upper hybrid oscillations trapped in density irregularities in the ionosphere, Phys. Plasmas, 10, 2962-2970, 2003.

Kelley, M. C., Arce, T. L., Salowey, J., Sulzer, M., Armstrong, W. T., Carter, M., and Duncan, L.: Density depletions at the 10$\mathrm{m}$ scale induced by the Arecibo heater, J. Geophys. Res., 100, 17367-17376, 1995.

Kosch, M. J., Rietveld, M. T., Hagfors, T., and Leyser, T. B.: Highlatitude HF-induced airglow displaced equatorwards of the pump beam, Geophys. Res. Lett., 27, 2817-2820, 2000.

Kosch, M. J., Rietveld, M. T., Yeoman, T. K., Cierpka, K., and Hagfors, T.: The high-latitude artificial aurora of 21 February 1999: An analysis, Advances Polar Upper Atmos. Res., 16, 112, 2002a.

Kosch, M. J., Rietveld, M. T., Kavanagh, A. J., Davis, C., Yeoman, T. K., Honary, F., and T. Hagfors: High-latitude pump induced optical emissions for frequencies coose to 
third electron gyro-harmonic, Geophys. Res. Lett., 29, 2112, doi:10.1029/2002GL015744, 2002b.

Kosch, M. J., Pedersen, T., Hughes, J., Marshall, R., Gerken, E., Senior, A., Sentman, D., McCarric, M., and Djuth, F. T.: Artificial optical emission at HAARP for pump frequencies near third and second electron gyro-harmonic, Ann. Geophys., 23, 1585-1592, 2005 , http://www.ann-geophys.net/23/1585/2005/.

Kuo, S., Lee, M., and Kossey, P.: Excitation of oscillating two stream instability by upper hybrid pump in ionospheric heating experiments at Troms $\varnothing$, Geophys. Res. Lett., 24, 2969-2972, 1997.

Leyser, T. B., Gustavsson, B., Brändström, B. U., et al.: Simultaneous measurements of high frequency pump-enhanced airglow and ionospheric temperatures in auroral latitudes, Adv. Polar Upper Atmos. Res., 14, 1-11, 2000.

Leyser, T. B.: Stimulated electromagnetic emissions by high frequency electromagnetic pumping of the ionospheric plasma, Space Sci. Rev., 98, 223-328, 2001.

Mantas, G. P. and Carlson, H. C.: Reinterpretation of the 6300 A airglow enhancements observed in ionosphere heating experiments based on analysis of Plattevile, Colorado data, J. Geophys. Res., 101, 195-209, 1996.

Mishin, E. V., Carlson, H. C., and Hagfors, T.: On the electron distribution function in the $\mathrm{F}$ region and airglow enhancements during HF modification experiments, Geophys. Res. Lett., 27, 2857-2860, 2000.

Mishin, E. V., Burke, W. J., and Pedersen, T.: On the onset of HF-induced airglow at HAARP. J. Geophys. Res. 109, A02305, doi:10.1029/2003JA010205, 2004.

Myasnikov, E. N., Muravjeva, N. V., Sergeev, E. N., Frolov, V. L., Nasyrov, A. M., Nasyrov, I. A., Beley, V. S., Koloskov, A. V., Yampolsky, Yu. M., and Groves, K. M.: Spatial Spectrum of Artificial Ionospheric Irregularities Induced by Powerful HF Radiowaves. Radiophys. Quantum Electron, 44, 833-846, 2001.

Pedersen, T. R. and Carlson, H. C.: First observation of HF heater produced airglow at the High Frequency Active Auroral Research Program facility: Thermal excitation and spatial structuring, Radio Sci., 36, 1013-1026, 2001.

Pedersen, T. R., McCarric, M., Gerken, E., Selcher, C., Sentman, D., Carlson, H. C., and Gurevich, A. V.: Magnetic zenith enhancement of HF radio-induced airglow production at HAARP, Geophys. Res. Lett., 30, doi:10/1029/2002GL016096, 2003.

Perkins, F. W., Oberman, C. R., and Valeo, E. J.: Parametric instabilities and ionospheric modifications, J. Geophys. Res., 79, 1478-1483, 1974.

Perkins, F. W. and Valeo, E. J.: Thermal self-focusing of electromagnetic waves in plasmas, Phys. Rev. Lett., 32, 1234-1237, 1974.
Rietveld, M. T., Kosch, M. J., Blagoveshchenskaya, N. F., Kornienko, V. A., Leyser T. B., and Yeoman T. K.: Ionospheric electron heating, optical emission, and striation induced by poewrful HF radi0 waves at high latitudes: Aspect angle dependence, J. Geophys. Res., 108, 1141, doi:10.1029/2002JA009543, 2003.

Robinson, T. R.: The heating of the high latitude ionosphere by high power radio waves, Physics reports, 179, 79-209, 1989.

Sergeev, E. N. Grach, S. M., Komrakov, G. P., Frolov, V. L., Stubbe, P., Thidé, B., Leyser, T., and Carozzi, T.: Influence of smallscale irregularities on characteristics of the overshoot effect in the temporal evolution of stimulated electromagnetic emission. Part I. Development stage, Radiophys. Quantum Electron., Engl. Transl., 42, 544-556, 1999.

Sergeev, E. N., Grach, S. M., and Kotov, P. V.: Study of the excitation conditions and characteristics of ionospheric plasma turbulence at the development stage of the ponderomotive parametric instability, Radiophys. Quantum Electron, 47, 185-204, 2004.

Sergeev, E. N., Frolov, V. L., Grach, S. M., and Kotov, P.V.: On the morphology of SEE spectral features in a wide pump wave frequency range, Adv. Space Res., 38, 2518-2536, doi:10.1016/j.asr.2005.02.046, 2006

Strickland. D. J., Craven, J. D., and Daniell Jr., R. E.: Six days of thermosphric-ionospheric weather over the northern hemisphere in late September 1981, J. Geophys. Res., A106, 30 291-30306, 2001.

Tereshchenko, E. D., Khudukon, B. Z., Gurevich, A. V., Zybin, K. P., Frolov, V. L., Myasnikov, E. N., Muravieva, N. V., and Carlson, H. C.: Radio tomography and scintillation studies of ionospheric electron density modification caused by a powerful HF-wave and magnetic zenith effect at mid-latitudes, Phys. Lett., A325, 381-388, 2004.

Vas'kov, V. V. and Gurevich, A. V.: Resonance instability of smallscale plasma perturbations, Sov. Phys. J. Exp. Theor. Phys., 46, 487-494, 1977.

Vas'kov, V. V. and Gurevich, A. V.: Samofokusirovochnaya i resonansnaya neustoichvost' v F-oblasti v ionosfere, in: "Teplovye nelinienye effekty v plazme", 81-138, Gorky, 1979, (in Russian) (Self-focusing and resonant instability in the ionospheric $F$ region, in: "Thermal nonlinear phenomena in a plasma"), 1979.

Vlasov M. N., Kelley M. C., and Gerken, E.: Impact of vibrational excitation on ionoswpheric parameters and artificial airglow during HF heating in the $F$-region, J. Geophys, Res., 109, A093004, doi:10.1029/2003JA010316, 2004.

Weinstock, J. and Bezzerides, B.: Theory of electron acceleration during parametric instabilities, Phys. Rev. Lett., 32, 754-748, 1974. 